\title{
2020 Breakthrough Prize in Mathematics
}

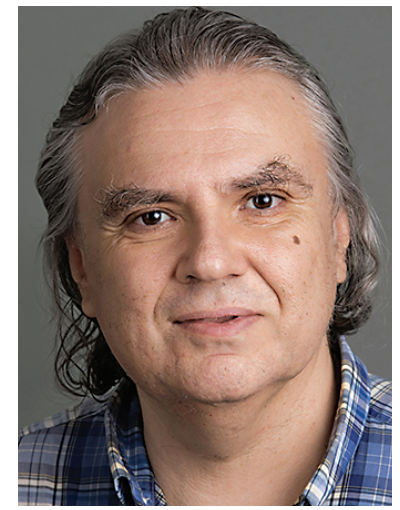

Alex Eskin
Alex Eskin of the University of Chicago has been awarded the 2020 Breakthrough Prize in Mathematics "for revolutionary discoveries in the dynamics and geometry of moduli spaces of Abelian differentials, including the proof of the 'magic wand theorem' with Maryam Mirzakhani." A description of this work reads: "Eskin teamed with famed Iranian mathematician and Fields Medalist Maryam Mirzakhani to prove a theorem about dynamics on moduli spaces. Their tour de force, published in 2013 after five years of labor, is a result with many consequences. One addresses the long-standing problem: If a beam of light from a point source bounces around a mirrored room, will it eventually reach the entire room-or will some parts remain forever dark? After translating the problem to a highly abstract multidimensional setting, the two mathematicians were able to show that for polygonal rooms with angles which are fractions of whole numbers, only a finite number of points would remain unlit." Mirzakhani passed away in 2017.

Alex Eskin was born in Moscow, USSR, in 1965. He received his PhD from Princeton University in 1993 under the direction of Peter Sarnak. He was a member of the Institute for Advanced Study from 1993 to 1994 before joining the faculty of the University of Chicago, where he has been Arthur Holly Compton Distinguished Service Professor since 2012. He has been the recipient of Alfred P. Sloan and NSF Postdoctoral Research Fellowships and of a Packard Fellowship for 1997-2002. He received the Clay Research Award in 2007 and a Simons Investigator Award in 2014. He has given invited talks at International Congresses of Mathematicians in Berlin in 1998 and in Hyderabad

For permission to reprint this article, please contact: reprint-permission aams.org.

DOI: https://dx.doi.org/10.1090/noti2032

in 2010. He is a Fellow of the AMS and a member of the National Academy of Sciences and the American Academy of Arts and Sciences.

The Breakthrough Prizes honor important, primarily recent, achievements in the categories of fundamental physics, life sciences, and mathematics. The prizes are sponsored by Sergey Brin, Priscilla Chan and Mark Zuckerberg, Pony $\mathrm{Ma}$, Yuri and Julia Milner, and Anne Wojcicki. The prize carries a cash award of US $\$ 3$ million.

In addition, three New Horizons in Mathematics Prizes were awarded for 2020 to promising early-career researchers. The prizes carry a cash award of US $\$ 100,000$. The prizes were awarded to the following.

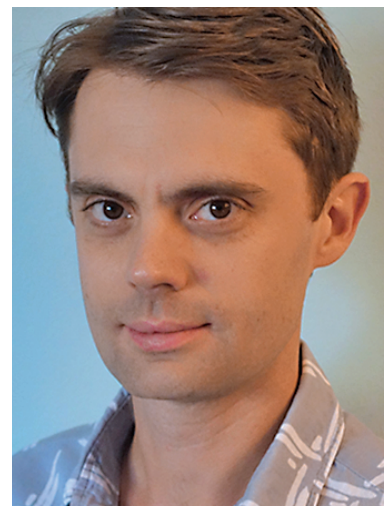

Tim Austin
Tim Austin of the University of California, Los Angeles, was honored for "multiple contributions to ergodic theory, most notably the solution of the weak Pinsker conjecture." Austin received his $\mathrm{PhD}$ from the University of California, Los Angeles, in 2010 under the direction of Terence Tao. Austin was named a Clay Research Fellow for the years 2010-2015. He held a visiting position at Brown University from 2010 to 2012, progressed from assistant to associate professor at the Courant Institute of Mathematical Sciences from 2012 to 2017, and joined the UCLA faculty in 2017. He has given a large number of talks, including the Bernoulli Lecture in Lausanne in 2019.

Emmy Murphy of Northwestern University received a New Horizons Prize for her "contributions to symplectic and contact geometry, in particular the introduction of notions of loose Legendrian submanifolds and, with Matthew Strom Borman and Yakov Eliashberg, overtwisted contact structures in higher dimensions." Murphy received her $\mathrm{PhD}$ from Stanford University in 2012 and was a member of the faculty of the Massachusetts Institute of Technology from 2012 to 2017. She joined Northwestern in 2016 and 


\section{COMMUNICATION}

has been an associate professor since 2018. She received a Von Neumann Fellowship at the Institute for Advanced Study for 2019-2020. She was awarded an Alfred P. Sloan Research Fellowship in 2015 and a Radcliffe Institute for Advanced Study Fellowship in 2016-2017. She received the AWM Birman Research Prize in Topology and Geometry in 2017 and was an invited speaker at the 2018 International Congress of Mathematicians.

Xinwen Zhu of the California Institute of Technology was awarded a New Horizons Prize "for work in arithmetic algebraic geometry including applications to the theory of Shimura varieties and the Riemann-Hilbert problem for p-adic varieties." He received his PhD from the University of California, Los Angeles, in 2009 under Edward Frenkel. He held positions at Harvard University and Northwestern University before joining Caltech in 2014. He was awarded an AMS Centennial Fellowship in 2013 and an Alfred P. Sloan Fellowship in 2015. In 2019 he was awarded the Morningside Gold Medal of Mathematics, given to exceptional mathematicians of Chinese descent under the age of forty-five.

-Elaine Kehoe from Breakthrough Prize announcements

Credits

Photo of Tim Austin is courtesy of Katherine Smith.


\section{engineering}

astronomy

robotics

genetcs

medicine

biology

clinatology

forensics.

statistics

finance

computer science

physics

neuroscience

chemistry

geOlOgy

ecology

molecular biology

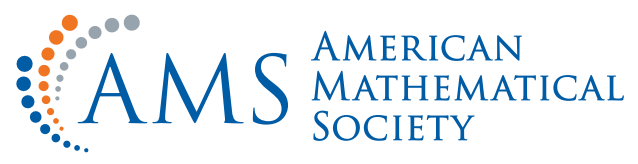

